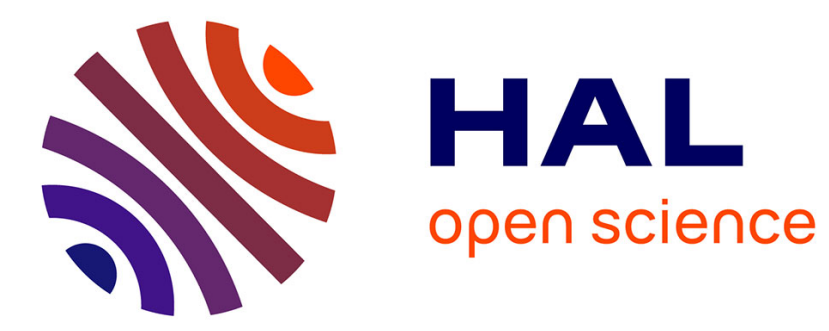

\title{
L'oiseau dans le géosystème. Essai de cartographie de l'avifaune dans le massif de Grésigne (Tarn)
}

Bernard Alet

\section{To cite this version:}

Bernard Alet. L'oiseau dans le géosystème. Essai de cartographie de l'avifaune dans le massif de Grésigne (Tarn). Revue Géographique des Pyrénées et du Sud-Ouest, 1986, 57 (3), pp.343-362. 10.3406/rgpso.1986.4953 . hal-02782172

HAL Id: hal-02782172

https://hal-univ-tlse2.archives-ouvertes.fr/hal-02782172

Submitted on 4 Jun 2020

HAL is a multi-disciplinary open access archive for the deposit and dissemination of scientific research documents, whether they are published or not. The documents may come from teaching and research institutions in France or abroad, or from public or private research centers.
L'archive ouverte pluridisciplinaire HAL, est destinée au dépôt et à la diffusion de documents scientifiques de niveau recherche, publiés ou non, émanant des établissements d'enseignement et de recherche français ou étrangers, des laboratoires publics ou privés. 


\section{L'oiseau dans le géosystème. Essai de cartographie de l'avifaune} dans le massif de Grésigne (Tarn)

\section{Bernard Alet}

\section{Citer ce document / Cite this document :}

Alet Bernard. L'oiseau dans le géosystème. Essai de cartographie de l'avifaune dans le massif de Grésigne (Tarn). In: Revue géographique des Pyrénées et du Sud-Ouest, tome 57, fascicule 3, 1986. L'élément et le système. pp. 343-362;

doi : https://doi.org/10.3406/rgpso.1986.4953

https://www.persee.fr/doc/rgpso_0035-3221_1986_num_57_3_4953

Fichier pdf généré le 06/04/2018 


\title{
Resumen
}

Las aves dentro del geosistema. Cartografía de la avifauna en el macizo de Grésigne (Tarn). El método geosistémico aplicado a la ornitología permite situar las poblaciones de aves en el espacio y en el tiempo así como conocer sus estrategias de aprovechamiento del medio ambiente, contribuyendo así a una cartografía integrada de geografía ornitológica. La principal dificultad es la de integrar las aves en determinados medios a causa de su movilidad y de sus exigencias espaciales muy variables según la estación. Por eso el mapa de las poblaciones sólo toma en cuenta las aves anidadoras. Después se compara este mapa de aves con el de los geosistemas. Resulta que no hay una, sino diversas avifaunas que coinciden esquemáticamente con los cuatro complejos geográficos puestos en evidencia por el mapa de los geosistemas. A la cuatro avifaunas principales se añaden otras locales que corresponden a habitats de poca extendidos o marginales. Cada población de aves es estudiada dentro de la avifauna a la que pertenece. Con el análisis geosistémico también se puede entender el impacto de la acción antrópica en la evolución de los geosistemas y de sus avifaunas. Las aves dan fe de esta evolución porque son sensibles a las modificaciones de su entorno vital. Cualquier geofaciés es un estado intermedio y provisional que explica el carácter efímero de las poblaciones de aves y también su carácter repetitivo en la Historia. De allí la necesidad de una permanente reactualización de la cartografía ornitogeográfica.

\begin{abstract}
Birds and the geosystem : mapping birdforms in the Grésigne Massif (Tarn). The reconstruction in space and time of bird populations, as well as their environment use strategies is made possible by the application to ornithology of the geosystemic method. This contributes to an integrated cartography of geographic ornithology. It is uneasy to link birds to a defined environment, due to their mobility and their changing seasonal spatial demands, except in spring, with reproduction ; therefore the population map only refers to nesting birds. The geosystem map and that of birds are then compared. There are several distinct avifauna, linked schematically to the four geographic complexes indicated by the map of geosystems. Other avifauna and local populations are linked to limited or marginal habitats. Each bird population being studied in relation to its avifauna, the geosystemic analysis allows to understand the consequences of human action on the evolution of geosystems and their avifauna. The birds, characteristic of a certain state of the geosystem, indicate this evolution. Each basic landscape structure constitutes an intermediate stage which explain the ephemeral nature of these bird populations, but also their respective nature in time. The ornitho-geographic cartography, which represents the bird population at a moment in space and time, must be regularly recreated in order to follow their evolution in space and time.
\end{abstract}

\section{Résumé}

La méthode géosystémique, appliquée à l'ornithologie, resitue les peuplements d'oiseaux dans l'espace et dans le temps et leurs stratégies d'utilisation du milieu ; elle peut contribuer à une cartographie intégrée de géographie ornithologique. La difficulté principale est d'intégrer les oiseaux dans un milieu défini, en raison de leur mobilité et de leurs exigences spatiales très changeantes au fil des saisons, sauf au printemps, en période de reproduction ; aussi la carte des peuplements ne prendelle en compte que les oiseaux nicheurs. La carte des géosystèmes et celle des peuplements d'oiseaux nicheurs sont ensuite comparées avec leurs techniques respectives de réalisation. II existe plusieurs avifaunes correspondant aux quatre complexes géographiques mis en évidence. A ces quatre grandes avifaunes, s'ajoutent des avifaunes ou peuplements localisés, liés à des habitats peu étendus ou marginaux. Chaque peuplement d'oiseaux est étudié dans son avifaune. L'analyse géosystémique permet de comprendre l'effet et les conséquences de l'action anthropique sur l'évolution des géosystèmes et de leur avifaune. Les oiseaux, caractéristiques d'un état du géosystème à un moment donné, sont des témoins de cette évolution et sont à ce titre des indicateurs fiables parce que sensibles aux transformations de leur milieu de vie. Tout géofaciès constitue un stade intermédiaire, provisoire, qui explique le caractère éphémère des peuplements d'oiseaux mais aussi leur caractère répétitif au cours du temps. La cartographie ornitho-géographique, représentant les peuplements d'oiseaux à un moment donné, dans un espace donné, doit être réactualisée pour suivre l'évolution de ces peuplements dans l'espace et dans le temps. 


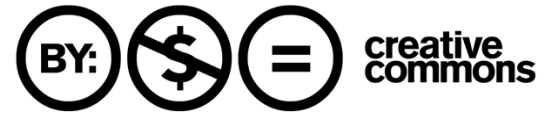


RIEVULE GEOGRAPHIOUI: DIES PYRENIFS LE DC SUID-OUEST

TOMF: 57, FAsc. 3, pp. 343-362, Toulouse, 1986.

\title{
L'oiseau dans le géosystème. Essai de cartographie de l'avifaune dans le massif de Grésigne (Tarn)*
}

\author{
par Bernard Alet *
}

\begin{abstract}
Le géosystème permet de resituer dans l'espace et dans le temps un processus ou un élément particulier du milieu. Pour être pleinement efficace, il doit être "finalisé »- ici, sur l'oiseau et ses stratégies d'utilisation de l'espace - et peut ainsi contribuer à une cartographie intégrée de géographie ornithologique (Alet, 1984 a).
\end{abstract}

\section{La méthode géosystèmique appliquée à l'ornithologie}

En ornithologie, le problème principal est la difficulté d'intégrer dans un milieu défini les oiseaux qui, du fait de leur mobilité et de leurs exigences spatiales aussi bien horizontales que verticales, très changeantes au fil des saisons, ne peuvent être "utilisés " pour caractériser des milieux trop exigus. En outre, l'éclectisme alimentaire, voire l'opportunisme de nombreux oiseaux et leur souplesse d'adaptation à des milieux très divers font que chaque espèce est rarement liée à un seul milieu. Ne peuvent donc être considérés comme bioindicateurs que les peuplements d'oiseaux et non les espèces prises isolément. Enfin, les oiseaux n'intègrent pas à eux seuls l'ensemble des données écologiques d'un milieu donné. Ces limites rendent toute cartographie de l'avifaune aléatoire, sauf au printemps, en période de reproduction, où les relations entre les oiseaux et leur milieu sont

(*) Planche hors-texte en couleurs.

(**) Chargé d'études au Conseil scientifique régional de l'environnement et CIMA-UA 366, CNRS. 
les plus étroites. En effet, pour mener à bien la nidification, les passereaux, en particulier, défendent contre toute intrusion des individus de la même espèce, leur territoire qui les fixent alors momentanément à leur milieu.

En raison de leur mobilité spatio-temporelle et des exigences écologiques de chaque espèce, les oiseaux s'insèrent à différents niveaux scalaires dans le système choro-taxonomique constitué par les unités territoriales. Il peut donc y avoir autant d'avifaunes que de niveaux d'échelle. Les espèces de grande taille, tels les rapaces, peuvent avoir un territoire chevauchant plusieurs géosystèmes; d'autres vivent dans les limites du géosystème ou du géofaciès, d'où l'importance des écotones pour toutes ces espèces. Celles-ci possèdent souvent un génotope (1) et un trophotope (2) distincts, situés de part et d'autre de l'écotone (3). Par ailleurs, le trophotope de chaque espèce change souvent au cours de l'année, en fonction de la phénologie des végétaux.

La mise en évidence d'unités spatiales, indépendantes par rappori aux stratégies d'utilisation des oiseaux, permet de mieux comprendre l'écologie de ces derniers, leur insertion dans un système défini, leur place par rapport aux autres espèces animales et autres éléments du milieu. La délimitation de l'aire maximale (domaine vital des oiseaux) et des aires spécialisées (génotope, trophotope) en est facilitée.

En tant qu'unité spatiale divisée en géofaciès et en géotopes, selon la définition classique (Bertrand, 1968, 1978), le géosystème est utilisé ici comme une simple "unité opérationnelle" pouvant être facilement cartographiée. D'où l'intérêt et l'utilité pratique de la carte des géosystèmes pour représenter rapidement les peuplements d'oiseaux en Grésigne. L'analyse phytogéographique seule n'est pas suffisante pour cartographier ces peuplements. En effet, "l'évaluation zooécologique d'un territoire et sa cartographie peuvent différer sensiblement de son évaluation phytoécologique " (Hansson, 1977). Le géosystème ne doit pas être considéré seulement comme une "unité naturelle territoriale "; il constitue surtout un "complexe " spatiotemporel dans lequel s'insère une biocénose et notamment l'avifaune; il doit être assimilé à un tout, vivant, en perpétuelle évolution dans l'espace et dans le temps, non seulement à l'échelle historique, mais aussi au cours de l'année, et même au cours de la journée. Ce complexe spatial est doté d'une structure, d'un fonctionnement et d'un comportement propres, mais ne doit pas être isolé des systèmes voisins. Les géosystèmes ont en effet un rôle de complémentarité pour les oiseaux, notamment dans leur rythme biologique annuel, au cours duquel ils changent fréquemment de sites d'alimentation, d'où l'importance reconnue au concept d'écotone.

Toutefois, si chaque géosystème possède une dynamique et une évolution propres, il ne présente pas nécessairement une grande homo-

(1) Site de reproduction.

(2) Site d'alimentation.

(3) Zone de transition entre deux milieux (lisière). 
généité physionomique, puisqu'on y trouve de nombreux stades d'évo. lution. Ce sont donc les géofaciès, unités territoriales homogènes, qui correspondent au peuplement d'oiseaux, ceux-ci étant plus sensibles à l'architecture de la végétation qu'à sa composition floristique. D'unc part, le géofaciès impose une structure au peuplement d'oiseaux; d'autre part, celui-ci n'est pas une entité définie, fermée, pouvant vivre en autarcie. En effet, il n'y a pas de frontière nette entre les peuplement d'oiseaux mais au contraire des interpénétrations permanentes. Les différentes espèces évoluent, s'adaptent et s'ajustent pour former un ensemble cohérent ayant une structure et un métabolisme propres (Blondel, 1979). Le peuplement n'est donc pas un assemblage fortuit d'espèces : il correspond à un niveau d'organisation dans lequel ces espèces utilisent de façon complémentaire les ressources offertes par le géofaciès (sites de nidification, nourriture, abris). Chaque peuplement d'oiseaux peut donc être caractérisé, en première approximation, par la physionomie de la végétation, depuis les milieux fermés, pluristrates et denses jusqu'aux milieux ouverts monostrate ou sans strate. Hauteur et densité de la végétation sont ainsi deux éléments déterminants du peuplement d'oiseaux.

Si le géofaciès détermine la répartition horizontale des oiseaux, le géohorizon détermine leur répartition verticale. Il constitue d'une part le trophotope des oiseaux et, d'autre part, lcur génotope, à la période de reproduction. Le géohorizon a d'autres rôles : abri, reposoir, dortoir... Il correspond non seulement à la strate de végétation, - cas d'utilisation le plus fréquent pour les oiseaux -, mais aussi à l'ensemble des composants du géofaciès (air, eau, sol, etc.), auxquels sont liées certaines espèces.

Cependant, le géohorizon constitue un cadre trop rigide. En effet, le découpage en compartiments se superposant les uns aux autres (géohorizons) a pour inconvénient de sectionner le "cadre de vie» de l'oiseau : les troncs d'arbres, auxquels sont liés les pics par exemple, ne sont pas des géohorizons, mais seulement des éléments caractéristiques de géohorizons.

\section{La cartographie ornitho-géographique}

La cartographie dont les buts, ainsi que les méthodes et techniques d'échantillonnage de l'avifaune en Grésigne, ont été brièvement exposés (Alet, 1984 b), porte surtout sur les oiseaux nicheurs, puisque c'est au printemps qu'ils sont le plus étroitement liés à leur support végétal. Aux autres saisons, notamment en automne et en hiver, la répartition des oiseaux est difficile à cartographier, car elle dépend surtout de la quantité de nourriture disponible et des conditions des types de temps. Les oiseaux, même les sédentaires, deviennent plus ou moins erratiques et se dispersent dans tous les milieux qui leur sont momentanément favorables. 
La cartographie ne représente donc les peuplements d'oiseaux nicheurs quà un moment donné (printemps 1982), dans un espace donné. Nous avons vu, en outre, que certaines espèces ayant un vaste rayon d'action ou un territoire étendu, ont un génutope et un trophotope distincts, d'où l'importance pour elles des écotones, mais aussi la difficulté de cartographier leur répartition, à cheval sur plusieurs milieux. Ainsi, la carte des peuplements d'oiseaux nicheurs ne prendelle en compte que les oiseaux dont génotope et trophotope sont confondus; c'est notamment le cas des passereaux. Sur les 102 espèces nicheuses que comptait l'avifaune de Grésigne au printemps 1982, environ 40 sont des ubiquistes pouvant habiter des milieux très divers et s'intégrer dans la plupart des géosystèmes; au contraire, une soixantaine d'entre elles sont localisées à des milieux bien définis. Ce sont donc ces espèces bio-indicatrices qui déterminent chaque peuplement d'oiseaux caractéristique d'un milieu.

Pour comprendre la répartition actuelle de l'avifaune en Grésigne, il faut se situer à plusieurs niveaux d'analyse en réduisant progressivement l'échelle de perception du secteur étudié, depuis l'échelle "continentale», expliquant l'origine et la composition de l'avifaune, jusqu'au géohorizon, en passant par l'échelle régionale (4), le géosystème et le géofaciès. Ce système gigogne permet une analyse multidimensionnelle de l'avifaune qui reste encore grossière mais n'avait jamais été tentée du point de vue cartographique.

\section{Les géosystèmes de Grésigne et leur avifaune.}

Le massif de Grésigne, environ 15600 hectares, est à la charnière de trois régions naturelles, dont le nœud est constitué par la forêt domaniale de Grésigne. Les différents milieux qui les composent sont, à des degrés divers, artificialisés et insérés dans des systèmes de production. C'est cette typologie, à partir du système de production dominant, qui sera adoptée pour distinguer et cartographier les quatre complexes et leurs avifaunes respectives. Ces quatre complexes sont donc issus du regroupement de géosystèmes voisins.

a). La forêt domaniale de Grésigne comporte trois géosystèmes $\left(G_{1}, G_{2}, G_{3}\right)$ qui forment le premier complexe spatio-temporel : espace entièrement aménagé pour la production de bois d'œuvre et surtout de pâte à papier, secondairement utilisé comme espace cynégétique et touristique. A ce premier complexe seront rattachés les taillis denses de bordure pour obtenir un premier ensemble ornithologique, celui qui correspond à l'avifaune forestière.

b). Le géosystème du Ségala de Vaour $\left(\mathrm{G}_{\mathbf{4}}\right)$ dépend des Ségalas rouergat et tarnais, vastes plateaux bocagers du sud-ouest du Massif Central, spécialisés dans l'élevage bovin (production laitière et de veaux de boucherie). A ce géosystème est rattaché celui de la dépres-

(4) Cf. carton de la carte des peuplements d'oiseaux nicheurs. 
sion bocagère de Penne $\left(G_{i}\right)$ pour former un deuxième complexe auquel correspond l'avifaune du bocage et des milieux mixtes aux écotones multiples.

c). Les géosystèmes des plateaux calcaires de Magrou et des Abriols $\left(G_{\text {; }}\right.$ et $\left.G_{3}\right)$ font partie de l'extrémité sud du Causse de Limogne (Quercy) : espace agro-pastoral en voie d'abandon, économiquement marginalisć et qui a connu un fort exode rural, où la production est tournée vers l'élevage ovin. Ces géosystèmes forment un troisième complexe auquel correspond l'avifaune des friches et des causses en voic d'abandon.

d). Trois géosystèmes agricoles $\left(G_{1}, G_{*}\right.$ et $G_{11}$ ) appartiennent aux côteaux molassiques du Gaillacois et, plus généralement, de l'Albigeois et du Montalbanais : espace céréalier et viticole (vignoble du Gaillacois) formant un quatrième complexe avec une avifaune lièe aux milieux cultivés.

Chacune des trois régions évoquées, et la forêt domaniale elle-même, apportent leur «contingent » d'espèces caractéristiques, ayant plus ou moins les mêmes affinités écologiques et les mêmes réactions (adaptation ou non) face aux interventions humaines. Nous n'étudierons donc pas une mais quatre grandes avifaunes correspondant schématiquement aux quatre complexes géographiques ainsi mis en évidence. Il s'agira de comparer entre elles la carte des géosystèmes et la carte des peuplements d'oiseaux nicheurs et de mettre en évidence les techniques respectives de réalisation.

La plupart des limites retenues sont identiques pour les deux cartes. C'est le cas de tous les taillis, des enrésinements, des cultures et des vignobles, des landes acidophiles, des coupes rases et des fourrés en forêt domaniale; c'est aussi le cas de la futaie, limitée à la forêt domaniale. Ce sont surtout la couleur et la trame qui diffèrent d'une carte à l'autre, selon ce que l'on veut représenter - ici, les peuplements d'oiseaux nicheurs - La gamme chromatique utilisée en phytogéographie depuis $H$. Gaussen a été transposée à l'avifaune : chaque couleur a une signification "globale " non seulement écologique mais aussi socio-économique; elle caractérise donc chaque complexe ainsi que l'avifaune correspondante.

\section{La carte des géosystèmes de Grésigne.}

Sur cette carte, la couleur correspond à la fois aux éléments physiques (relief, micro-climat), bio'ogiques (végétation, surtout) et socio-économiques dominants de chaque géosystème. En revanche, elle n'indique pas la physionomie de la végétation. La trame permet de caractériser chaque géofaciès: elle a une signification physionomique et représente la structure verticale du géofaciès; celui-ci indique non seulement la végétation correspondante, mais aussi l'évolution historique de cette couverture végétale (depuis un siècle environ) due pour une large part à l'action anthropique. Pour rendre cette carte 
plus claire, tous les stades d'évolution des géofaciès ne sont pas indiqués, alors que ces différents stades sont souvent déterminants dans la répartition des oiseaux; mais ils sont signalés clans le texte.

Chaque géofaciès évolue à son propre rythme et ses stades d'évolution correspondent à un certain état de la végétation à un moment donné. Cet état, qui est la plupart du temps transitoire, constitue la physionomie du géofaciès. La prise en compte du facteur anthropique est fondamentale pour expliquer les mutations historiques des géofaciès et leurs états successifs, donc leur physionomie.

La carte dcs gćosystèmes résulte d'une analyse globale, intégrée, de l'espace grésignol. Chaque géofaciès est cartographié par une plage homogène, soit en aplat, soit en trame: l'intérêt de cette techniquc cartographique est précisément d'éviter toute surcharge et de rendre la carte des géosystèmes plus lisible. Une seule exception à cette règle : les enrésinements; afin de montrer le caractère artificiel récent des plantations de résineux qui affectent la majorité des géosystèmes, ces enrésinements représentés par des triangles ont été simplement superposés aux géofaciès antérieurs. Toutefois, cette carte géosystémique a des limites; en effet, les géosystèmes, en tant qu'unités spatiales horizontales, n'intègrent pas tous les éléments à prendre en compte pour l'étude de l'avifaune. Ainsi, la structure verticale n'apparaît-elle pas: les parois calcaires, les géohorizons, par exemple. En outre, les milieux linéaires (ripisylves, cours d'eau) et ponctuels (constructions humaines), fondamentaux pour les oiseaux, doivent être mis en évidence, au même titre que la physionomie de la végétation.

\section{La carte des peuplements d'oiseaux nicheurs au printemps 1982.}

Aux quatre grandes avifaunes déjà citées et largement répandues en Grésigne, s'en ajoutent d'autres spatialement plus limitées, liées à des habitats très spécialisés, peu étendus ou marginaux : avifaunes rupestre (liée aux parois calcaires des gorges), "synanthrope " (liée aux surfaces bâties), landicole, aquatique (mares et cours d'eau), etc., qui sont en fait des peuplements particuliers correspondant à des géofaciès qui n'avaient pu être distingués sur la carte des géosystèmes. Il s'agit, dans tous les cas, soit de peuplements d'oiseaux ponctuels (bourgs et hameaux, landes, etc.), soit de peuplements linéaires (cours d'eau et leurs ripisylves, haies, lisières, parois calcaires, etc.). D'une façon générale, la couleur représente les différentes avifaunes du massif de Grésigne, sauf cas particuliers pour bien mettre en évidence des espèces ou des peuplements d'oiseaux bio-indicateurs, ou bien des espèces très localisées dans le massif et donc rares. Ainsi les points violets indiquent-ils le peuplement particulier des landes acidophiles des croupes de Vaour avec la fauvette pitchou (Sylvia undata), la couleur rouge correspond au peuplement des chênes verts et pubescents avec notamment la fauvette passerinette (S. cantillans); la 
couleur bleue désigne, en forêt domaniale, le peuplement de la haute fûtaie plus ou moins claire et distingue des espèces inféodées à la vieille chênaie-hêtraie des grands versants humides.

La couleur correspondant à chaque avifaune, la trame indique les différents peuplements d'oiseaux, c'est-à-dire des groupes d'espèces présentant les mêmes exigences écologiques ou des exigences voisines, et ayant la même "réponse " face aux modifications de leur habitat. Les oiseaux ont ainsi été rassemblés en 21 peuplements définis après regroupement des géofaciès présentant une grande similitude physionomique. Mais seule apparaît la répartition horizontale des oiscaux qui ont généralement un génotope et un trophotope communs. Afin de cartographier la répartition des espèces en fonction de la structure verticale du géofaciès, on a situé le génotope des 60 espèces les plus caractéristiques dans leurs géohorizons respectifs (sol, strates de végétation...) codés de 0 à 5 , avec une mention particulière pour les espèces dites "cavicoles " car nichant dans des cavités : 6. Chaque peuplement d'oiseaux est caractérisé par deux éléments déterminants : la hauteur de la végétation et sa densité (recouvrement approximatif de chaque strate de 0 à $100 \%$ ).

Nous avons dressé une échelle indiquant la physionomie de la végétation, à partir de laquelle les 21 peuplements d'oiseaux ont été regroupés et classés. Cette śchelle physionomique part des formations végétales hautes et fermées (4 et 5 strates) indiquées en aplat, caractérisant les peuplements $P 1$ à $P 7$, jusqu'aux formations très ouvertes (monostrate ou sans strate) indiquées par des pointillés et tiretés, caratérisant les peuplements P8, P18 et P19, en passant par toute une série de milieux intermédiaires ( $P 9$ à $P 13$ ). Les milieux divers, ponctucls ou linćaires, sont également représentés par des symboles (P15, P16, P20 et P21). Nous savons que cette physionomic dépend presque toujours de l'action humaine: degré d'abandon des parcelles, stade d'évolution des traitements forestiers, plantations de résineux ou de peupliers, remembrement modifiant le narcellaire et la physionomie de la végétation, recalibrage des cours d'eau modifiant la structure des ripisylves...

Deux classifications des peuplements d'oiseaux ont été adoptées dans la légende de la carte:

- d'une part, en ce qui concerne leur répartition verticale en fonction des géohorizons, ils ont été regroupés au sein de leurs avifaunes respectives,

- d'autre part, en ce qui concerne leur répartition horizontale, ils ont été regroupés sur une même échelle. selon une séquence moyenne, en fonction de la physionomie des géofaciès.

Enfin, cette carte des peuplements d'oiseaux indiaue non seulement les différents stades d'évolution des milieux en 1982, mais aussi la nature de leurs écotones et le sens de l'évolution de ces derniers. En effet, nous avons distingué les lisières nettes, franches, bien marquées des lisières ménagées ou peu nettes, ainsi que la vitesse (rapide, 
lente ou nulle) et le sens d'évolution de tel milieu (fronts pionniers forestiers ou friches conquérantes). On peut donc sinon prévoir des scénarios d'évolution des peuplements d'après les états successifs des géofaciès, du moins mettre un peu de "dynamique » à la fois dans les paysages et dans les peuplements d'oiseaux.

a. L'avifaune forestière (couleurs dominantes verte et bleue) doit être divisée en deux grands peuplements distincts, liés l'un à la futaie, l'autre aux taillis de bordure.

Peuplements d'oiseaux liés à la futaie. Il s'agit de la forêt domaniale de Grésigne, traitée en futaie depuis 1875. Le peuplement de cette futaie est caractérisé par les couleurs bleu et vert sombre. La limite entre les deux grands peuplements de la futaie et des taillis correspond donc à la limite administrative de la forêt domaniale, sauf quelques parcelles récemment acquises par l'ONF et encore en taillis. Par contre, les géofaciès cartographiés sur la carte des géosystèmes ne correspondent plus aux limites des peuplements (et viceversa), sauf les coupes rases, les fourrés et les enrésinements qui possèdent des oiseaux caractéristiques. La distinction fondamentale entre les deux cartes est que les différents peuplements sont calqués non plus sur la composition floristique des parcelles (essences...) mais sur les modes de traitements forestiers, liés à la sylviculture: par exemple, traitement en futaie claire, futaie à sous-étage...

Le peuplement P1 (bleu) est constitué d'espèces «septentrionales», peu communes dans le sud-ouest de la France (mise à part la chaîne pvrénéenne) et inféodées aux vieilles futaies plus ou moins claires de chênes et de hêtres des grands versants. Ces futaies ont souvent plus de 150 ans d'âge et peuvent même dépasser 200 ans. Les espèces caractéristiques de ce milieu sont le pouillot siffleur, le pic mar, la mésange nonnette, la bécasse... Ce peuplement correspond à l'ensem. ble des hautes futaies des grands versants, puisque l'on y retrouve les mêmes espèces d'oiseaux. Le bleu foncé, indiquant la hêtraie, n'a pas été utilisé pour cette carte. En effet, il n'y a pas de peuplement spécifique de la hêtraie, qui est, en outre, beaucoup trop réduite aujourd'hui pour jouer un rôle dans la colonisation par certaines espèces plus ou moins inféodées au hêtre comme le pic noir (Dryocopus martius).

Le peuplement P3 de la futaie à sous-étage (vert sombre), où tous les géohorizons sont représentés, renferme de nombreuses espèces ubiquistes parmi les turdidés, les sylviidés, les paridés... En revanche, le peuplement P2 (vert clair) correspond à celui de la futaie éclaircie. caractérisée par l'absence de sous-bois. Entre ces deux peuplements $\mathrm{P} 2$ et $\mathrm{P} 3$, il n'y a pas de différence significative du nombre d'espèces, mais seulement une diminution de la fréquence de celles qui sont inféodées au sous-bois : c'est le cas par exemple, parmi les sylviidés, de la fauvette à tête noire et du pouillot véloce, mais aussi du troglodyte.

En forêt domaniale, la distinction entre les géofaciès de versants 
et de fonds de vallons larges ou étroits n'est pas nécessaire. Ce n'est pas un facteur déterminant dans la répartition des oiseaux. De même, les secteurs à Phillyrea media n'ont pas été indiqués car ils n'ont pas de peuplement spécifique. Ces secteurs secs de la cuvette centrale, où se mêlent les chênes pubescents et les chênes hybrides et qui peuvent être assimilés à des taillis, n'ont pas pu être distingués, car ils sont très diffus, mal délimités et donc peu cartographiables.

Peuplements d'oiseaux liés aux taillis denses. Ces taillis plus ou moins denses, sur les "serres » de conglomérats (G8), sur le plateau du Ségala de Vaour (G4) ou dans la dépression de Penne (G5), renferment un peuplement identique (P4). Celui-ci diffère peu du peuplement de la futaie à sous-étage en forêt domaniale, si ce n'est précisément par l'absence des espèces inféodées à la futaie, comme par exemple le pic mar, le pouillot siffleur, le gros-bec... Les taillis de châtaigniers, n'ayant pas d'espèce caractéristique, ne figurent pas sur la carte des peuplements d'oiseaux.

Peuplements d'oiseaux liés aux enrésinements de plus de 10 ans d'âge. Tous les reboisements en résineux, en forêt domaniale ou à sa périphérie, ont un peuplement d'oiseaux identique; ils ont donc été regrroupés. Mais leur âge a été distingué, d'une façon un peu arbitraire, afin de tenir compte du stade de croissance (stade physionomique) des plantations, au moment de cette étude (1982).

D'une part, les enrésinements de moins de 10 ans: effectués, en général, sur des coupes rases en forêt domaniale, colonisées par la lande à sarothamne ou sur les landes acidophiles à bruyères des croupes de Vaour; dans les deux cas, nous avons constaté que ces reboisements récents du fait de leur physionomie ont encore une avifaune caractéristique "landicolc", ct sont donc provisoirement assimilés par les oiseaux à des landes.

D'autre part, les enrésinements de plus de 10 ans, qui ont atteint une hauteur de plus de $5 \mathrm{~m}$ et ont fermé l'espace constitué par les landes, sont progressivement colonisés par un peuplement d'oiseaux particulier (P5), lié aux conifères; les espèces les plus caractéristiques de ce milieu sont le hibou moyen-duc, uniquement pour son génotope, la mésange noire, le roitelet triple-bandeau, le bouvreuil.

b. Avifaune des friches et des causses en voie d'abandon (couleur dominante jaune). Cette avifaune caractérise tous les milieux en voie d'enfrichement et de fermeture, généralement sur terrains secs et chauds, quels que soient les géosystèmes. Ainsi, les deux géosystèmes des causses, de même que les géofaciès g91 et g92 des coteaux marnocalcaires viticoles ont-ils été regroupés sous la même couleur, puisqu'ils ont une avifaune identique que l'on peut qualifier " avifaune des friches ".

Les peuplements $\mathrm{P} 6$ et $\mathrm{P} 7$ sont très voisins du peuplement $\mathrm{P} 4$ des taillis denses. On y retrouve, en effet, de nombreuses espèces forestières. La différence fondamentale est que les taillis de chênes pubescents sur les plateaux calcaires des causses sont des taillis clairs ou 
peu denses. On note, par conséquent, dans ces taillis des espèces caractéristiques des milieux ensoleillés, secs et chauds, telles que le pouillot de Bonelli, le circaète à un degré moindre... (peuplement P6). La couleur rouge a été conservée, par rapport à la carte des géosystèmes, pour indiquer la présence originale de la fauvette passerinette; sa répartition se calque sur le géofaciès 71 des taillis de chênes verts et pubescents en exposition sud (peuplement P7).

En revanche, les différents stades d'enfrichement ont été distingués sur la carte des peuplements d'oiseaux. Nous passons ainsi successivement du peuplement P8 de la pelouse-lande à brachypode (avec des espèces caractéristiques, tels l'alouette lulu, le pipit rousseline, le traquet pâtre, le bruant ortolan) au peuplement $P 9$ de la lande à fruticées (avec l'engoulevent, la perdrix rouge, la pie-grièche écorcheur, la linotte mélodieuse), puis à celui de la lande arborée P10 (avec la tourterelle des bois, le pipit des arbres, le pouillot de Bonelli...), enfin aux peuplements P6 et P7 des taillis, qui ont été décrits plus haut. C'est la trame qui distingue ici les différents stades d'enfrichement correspondant aux peuplements.

c. Avifaune du bocage et des milieux mixtes aux écotones multi. ples (couleur dominante vert tendre). Les deux géosystèmes du 'Terrefort de Penne (G5) et du Ségala de Vaour (G4), malgré des différences géomorphologiques et économiques, présentent des couvertures végétales dont les physionomies sont à peu près identiques : milieux bocagers, où les prairies alternent avec les haies vives. Ils ont la même avifaune et ont donc été regroupés sous la même couleur. Le bocage ne renferme pas d'espèces caractéristiques, mises à part quelques-unes que l'on retrouve plus régulièrement: la chouette chevêche, le pic vcrt, la huppe, le bruant proyer... En revanche, s'y rencontrent de nombreuses espèces souvent ubiquistes, issues des milieux fermés (taillis, forêt domaniale) et ouverts (prairies, cultures). C'est une avifaune très complexe, riche en espèces. Les fonds de vallons humides et les plateaux bocagers constituent le peuplement d'oiseaux P14.

d. Avifaune des milieux cultivés (couleur dominante marron). Ici aussi, les trois géosystèmes agricoles du sud-est (G8, G9 et G10) ont été regroupés. Les labours céréaliers et le vignoble ont été distingués malgré des peuplements d'oiseaux voisins. Le peuplement P18 qui correspond aux cultures céréalières comprend des espèces caractéristiques telles que la perdrix rouge, la caille, le busard cendré... Le peuplement P19 est lié au vignoble; il s'agit de la linotte mélodieuse, du brant ortolan... Ces deux milieux ont toutefois une avifaune commune, composée généralement d'espèces granivores comme de nombreux fringilles, bruants et moineaux, ainsi que du traquet pâtre, de l'alouette des champs. Le peuplement d'oiseaux des cultures céréalières $\mathrm{P} 18$ est le même quels que soient les géosystèmes et leurs géofaciès (par exemple, g74 dépressions karstiques des causses...).

e. Avifaunes ou peuplements d'oiseaux localisés. A ces quatre grandes avifaunes, il faut ajouter des avifaunes ou des peuplements plus 
spécialisés, correspondant à des milieux ponctuels ou linéaires qui n'apparaissent pas sur la carte des géosystèmes (sauf les landes et les peupleraies).

L'avifaune landicole (au sens strict) correspond, sur la carte des peuplements, aux points violets ou verts. En effet, ce sont généralement les mêmes espèces qui peuplent les différents types de landes (busard Saint Martin, fauvette grisette, hypolaïs polyglotte), mais il faut distinguer deux cas, selon que les landes se situent à l'intérieur ou à l'extérieur de la forêt domaniale de Grésigne. Dans le premiè cas, ce sont les coupes à blanc-étoc colonisćes par la lande à sarothamnes (P12); ici la fauvette pitchou est absente, car les coupes, localisées dans le centre de la forêt, relativement limitées dans l'espace et le temps, ne conviennent pas à cette espèce qui nécessite des horizons dégagés (points verts). Dans le deuxième cas, ce sont les landes acidophiles des croupes de Vaour (avec bruyère à balai, bruyère cendrée, callune) (P11); ici, au contraire, la situation et la physionomie des landes conviennent bien à la fauvette pitchou, espèce latéméditerranćenne originale en Grésigne (points violets).

Nous savons déjà que les enrésinements récents d'une partie dc: ces landes (moins de 10 ans) n'influent pas de façon significative sur la composition du peuplement, puisqu'ils sont encore provisoirement assimilés par les oiseaux à des landes. Par contre, lorsqu'ils seront plus développés, l'avifaune landicole sera alors remplacée par le peuplement des résineux P5.

Avec l'avifaune "svnanthrope » (couleur grise), il s'agit du peuplement P21, lié aux surfaces bâties : bourgs, hameaux, bâtiments divers, ruines... Les espèces les plus caractéristiques de ce peuplement sont la chouctte cffraie, la tourterelle turque, le pigeon biset semi-domestique, le martinet noir, les hirondelles de cheminće et de fenêtre, le rouge-queue noir, le moineau domestique.

L'avifaune de la movenne vallée remembrée de la Vère : le géofaciès: g101 des peupleraies et prairies humides en zone inondable correspond au peuplement P17. Les plantations de peupliers gagnent du terrain depuis le remembrement de la vallée de la Vère en 1972 et ce géofaciès se ferme très rapidement. Elles auront à moyen terme deux conséquences opposées pour deux peuplements d'oiseaux : d'une part, ces plantations favorisent l'extension du peuplement lié au géohorizon arboré (milan noir, pic épéichette, loriot): d'autre part, elles entraînent la disparition des espèces inféodées aux prairies plus ou moins humides : busard cendré, alouette des champs et, surtout, cisticole, oiseau méditerranéen peu répandu en Grésigne, dont l'extinction a été accélérée par les rigueurs de l'hiver 1985. Les peupleraies et prairies humides, en alternance, renferment donc deux peuplements d'oiseaux distincts, provisoirement indissociables. C'est pourquoi ceux-ci n'ont pu être cartographiés séparément.

L'avifaune rupestre, peuplement $\mathrm{P} 20$, est composée d'espèces très spécialisées dans leur habitat, liées notamment aux corniches et parois 
calcaires des gorges de l'Aveyron et de la basse vallée de la Vère. C'est le cas du faucon pélerin, du pigeon colombin, du martinet à ventre blanc, du choucas. du grand corbeau, du rouge-queue noir, de l'hirondelle des rochers.

L'avifaune des cours d'eau et de leurs ripisylves est composée d'espèces aquatiques inféodées aux cours d'eau : poule d'eau, martinpêcheur, bergeronnette des ruisseaux, cincle plongeur (ce derniel. sur le seul Aveyron), ainsi que les espèces liées aux ripisylves : milan noir, faucon hobereau, pic épeichette, loriot, fauvette dea jardins, rossignol: aui constituent deux peuplements distincts, respectivement $\mathrm{P} 16$ et $\mathrm{P} 15$, bien qu'ils soient toujours en combinaison et donc cartographiquement indissociables.

\section{Bilan provisoire.}

L'étude et la cartographie des quatre avifaunes dans leur complexe géographique respectif mettent en évidence deux séries de faits.

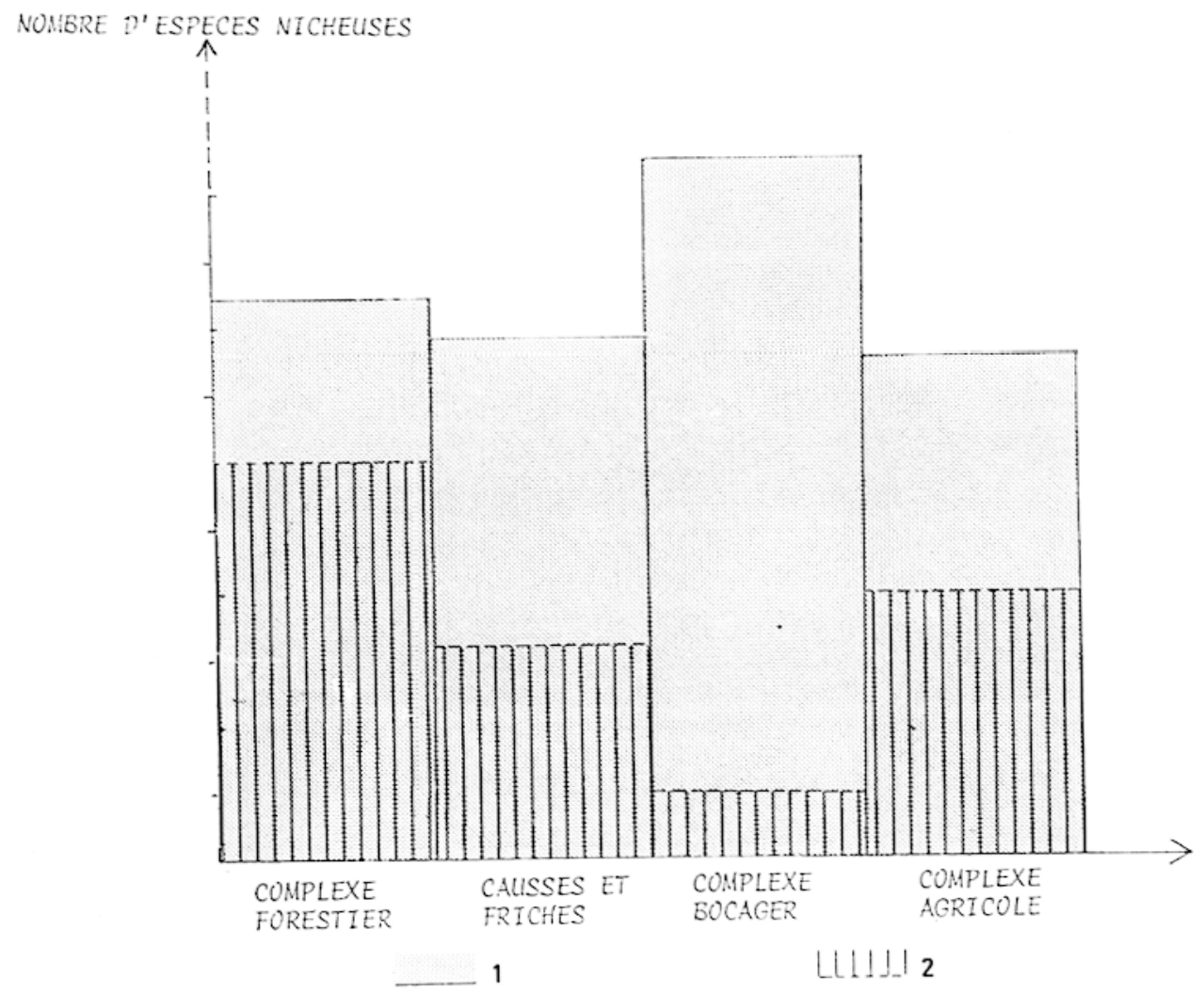

Fig. 1

Complexes et diversité des avifaunes de Grésigne. Essai de synthèse pour l'année 1982.

1. Richesse totale de l'avifaune. -2 . Espèces caractéristiques (approximations). (axe des ordonnées gradué de 5 en 5). 
Les espèces les plus caractéristiques sont celles des milieux « extrêmes ": d'un côté, une trentaine d'espèces typiquement forestières, liées aux formations ligneuses, hautes, pluristrates; de l'autre, une vingtaine d'espèces liées aux milieux ouverts, monostrates ou sans strate. Le nombre élevé des espèces forestières est en rapport étroit avec le nombre de géohorizons, donc avec la diversité des génotopes. Le nombre relativement élevé des espèces caractéristiques des milieux cultivés (oiseaux granivores) s'explique sans doute par la quantité de nourriture disponible et donc l'abondance des trophotopes. Les formations intermédiaires basses telles que les friches ont aussi leurs espèces bio-indicatrices, mais en moins grand nombre (environ une quinzaine), alors que les milieux mixtes bocagers en renferment peu. Enfin, ce sont les milieux linéaires ou ponctuels qui possèdent les espèces les plus spécialisées dans leur domaine vital (fig. 1).

Par contre, si l'on considère non plus le nombre d'espèces carac. téristiques mais la richesse spécifique totale de l'avifaune (Blondel, 1979), le complexe géographique ayant la plus grande diversité d'oiseaux est le complexe bocager aux écotones multiples avec environ 52 espèces. Le complexe forestier, relativement homogène, n'a qu'une quarantaine d'espèces (fig. 1). La richesse qualitative totale de l'avifaune d'un géosystème dépend donc non seulement du nombre de géohorizons (écotones internes horizontaux) mais aussi de la multiplicité des écotones fondamentaux (entre milieu ouvert et milieu fermé) c'est-à-dire de l'hétérogénéité du géosystème.

Le bocage de Grésigne est un complexe multipolaire, où les sources de nourriture et les sites de reproduction sont diversifićs. Selon Blondel, la diversité des oiseaux dans un tel milieu est grande, alors que lc nombre d'individus y est faible. Il y a donc un bon rapport entre prédateurs et proies; ceci explique que le bocage soit un complexe stable, en équilibre, contrairement aux complexes monopolaires (monocultures...) ayant un petit nombre d'espèces mais de nombreux individus (pullulations des espèces prolifiques liées à la rareté des prédateurs), donc instables, en désćquilibre.

\section{L'évolution des géosystèmes et de leur avifaune}

L'évolution des avifaunes dans l'espace et dans le temps s'accomplit, en gros, parallèlement à celle des géosystèmes, les oiseaux étant très sensibles aux modifications de leur milieu. L'analyse géosystémique présente un grand intérêt pour comprendre l'effet et les conséquences de l'action anthropique sur l'évolution des géosystèmes et donc de leurs avifaunes respectives. L'histoire des géosystèmes depuis plus d'un siècle est bien connue et cartographiée à l'échelle du 1/25.000 (Alet et al., 1980 et 1981). A partir de l'analyse géosystémique, on peut émettre plusieurs hypothèses sur les variations historiques de la répartition et de la composition des avifaunes de Grésigne. 
Celles-ci évoluent constamment, non seulement sous la pression des activités humaines, mais aussi sous la pression des facteurs physiques. biotiques et génétiques qui affectent les espèces. Certaines espèces régressent ou disparaissent, d'autres au contraire s'adaptent à l'action humaine et colonisent de nouveaux espaces. Les oiseaux sont non seulement des indicateurs écologiques mais aussi des intégrateurs de faits socio-économiques. C'est donc à partir du gradient d'anthropisation que les différentes avifaunes ont été analysées.

\section{TABleaU 1}

Evolition des MILIEUX ET DES PEUPLEMENTS d'OISEALX NICHEURS DEPUIS 30 ANS

\begin{tabular}{|c|c|c|c|c|c|}
\hline \multirow[t]{2}{*}{ EVOLUTION } & \multirow[t]{2}{*}{ DES MILIEUX } & \multicolumn{2}{|c|}{ GEOFACIES } & \multicolumn{2}{|c|}{$\begin{array}{l}\text { PEUPLEMENTS C'OISEAUX } \\
\text { NICHEURS }\end{array}$} \\
\hline & & En progression & En régression & En proaression & En régression \\
\hline \multirow[t]{2}{*}{$\begin{array}{l}\text { Progression lente } \\
\text { vers la fermeture }\end{array}$} & $\begin{array}{l}\text { Pronression des taillis denses sur } \\
\text { les prairies de fonds de vallons (1) }\end{array}$ & $\begin{array}{ll}9 & 4 \\
9 & 51 \\
0 & 81\end{array}$ & $\begin{array}{ll}5 & 47 \\
9 & 54 \\
9 & 84\end{array}$ & P 4 & P 14 \\
\hline & $\begin{array}{l}\text { Progression de la pelouse-lande sur } \\
\text { les cultures }\end{array}$ & & $\begin{array}{rr}0 & 62 \\
9 & 74 \\
- & -\end{array}$ & P 8 & P 18 \\
\hline \multirow[t]{5}{*}{$\begin{array}{l}\text { Procression rapide } \\
\text { vers la fermeture }\end{array}$} & $\begin{array}{l}\text { Progression des taillis clairs sur } \\
\text { les landes arborées }\end{array}$ & $\begin{array}{ll}9 & 61 \\
g & 72 \\
0 & 91\end{array}$ & \multirow{3}{*}{$\begin{array}{ll}9 & 63 \\
9 & 64 \\
9 & 73 \\
9 & 92\end{array}$} & \multirow{3}{*}{ 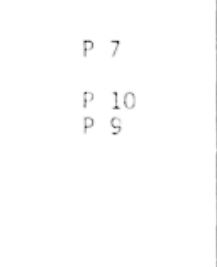 } & \multirow{3}{*}{$\begin{array}{l}\text { P } 10 \\
\text { P } 9 \\
\text { P } 8\end{array}$} \\
\hline & $\begin{array}{l}\text { Progression des landes arborées sur } \\
\text { les landes à fruticées }\end{array}$ & & & & \\
\hline & $\begin{array}{l}\text { Progression des landes à fruticées } \\
\text { sur pes pelouses-landes }\end{array}$ & & & & \\
\hline & Enrésinement des landes acidophiles & g 46 & S 45 & P. 5 & $P 11$ \\
\hline & Plantations de peupliers & 9101 & a 102 & $\begin{array}{c}\text { P } 17 \\
\text { peupliers } \\
-1\end{array}$ & $\begin{array}{c}\text { P } 17 \\
\text { prairies } \\
\text { humides }\end{array}$ \\
\hline \multirow{3}{*}{ Stabilité } & $\begin{array}{l}\text { Espace céréalier et viticole du sud- } \\
\text { est }\end{array}$ & \multicolumn{2}{|c|}{$983, g 84, g 93,994$} & \multicolumn{2}{|c|}{$P 18$ et $P 19$ stables } \\
\hline & $\begin{array}{l}\text { Terrefort de Penne et Ségala de } \\
\text { Vaour (bocage }\end{array}$ & \multicolumn{2}{|l|}{948,953} & \multicolumn{2}{|c|}{$P 14$ et $P 15$ stables } \\
\hline & $\begin{array}{l}\text { Landes acidophiles paraclimaciques } \\
\text { non enrésinées }\end{array}$ & \multicolumn{2}{|l|}{945} & \multicolumn{2}{|l|}{ P 11} \\
\hline
\end{tabular}

(1) Dynamique de végétation rapide naa is pression anthropique encore suffisante pour que l'évolution vers la fermeture soit lent

L'action anthropique se traduit de plusieurs façons, soit par un aménagement de l'espace, avec des transformations constructives ou destructives des géosystèmes (remembrement de la vallée de la Vère entraînant la suppression de haies, calibrage de la Vère avec disparition des ripisylves, changement d'assiette du vignoble du Gaillacois, modifications de certaines cultures et des rotations, sylviculture en forêt domaniale, enrésinements, populiculture...), soit par l'abandon des terroirs. Ces transformations des géosystèmes ont des conséquences sur la répartition des peuplements d'oiseaux (Tableau 1). 


\section{Effets de l'artificialisation et du morcellement des géofaciès sur les peuplements d'oiseaux.}

Dans le massif de Grésigne, les géofaciès forment une mosaïqu de milieux en perpétuelle évolution dans l'espace et dans le temps. Tout géofaciès constitue un stade intermédiaire, provisoire, de durée plus ou moins longue. Ceci explique le caractère éphémère des peuplements d'oiseaux, mais aussi leur caractère répétitif. Tout au long de l'évolution des géofaciès, les espèces d'oiseaux apparaissent, aug. mentent en densité, déclinent puis disparaissent à tour de rôle. De plus, chaque espèce évolue selon sa dynamique propre, indépendanment des autres, ce qui suggère un partage de l'espace tel que la compétition interspécifique soit réduite au maximum (Blondel, 1979). Les oiseaux se répartissent donc de façon complémentaire le long de la séquence d'évolution spatio-temporelle. Dans l'exemple de l'évolution des géofaciès des causses depuis la pelouse-lande jusqu'à la lande arborée et au taillis clair, on a montré qu'aucune espèce ne colonise la série complète, d'où un renouvellement total du peuplement d'oiseaux entre le premier et le dernier stade. Nous avons vu que les stades extrêmes contiennent le plus d'espèces spécialisées. En outre, le caractère éphémère des jeunes stades et la longévité des stades âgés signifient que le renouvellement des peuplements se ralentit le long des successions de végétation (Odum, 1969). D'où l'opposition existant entre des peuplements d'oiseaux pionniers, localisés et éphć. mères, et les peuplements stables occupant la plus grande partie des territoires.

On peut ainsi distinguer les espèces pionnières des cultures (passereaux granivores), des friches (fauvette grisette et hypolaiis polyglotte), des fronts arborés (pipit des arbres, tourterelle des bois, etc.). C'est, par contre, dans les vieux stades (vieille futaie) que les peuplements sont les plus stables, les oiseaux étant le plus souvent sédentaires. Les sites d'alimentation, variant considérablement dans l'espace au cours de l'année, sont utilisés par des espèces «vagabondes " grégaires. Ceci expliquerait la prolifération des passereaux granivores (fringilles et bruants) dans les milieux ouverts "jeunes» (Blondel, 1979).

Les traitements forestiers sont un bon exemple de l'évolution des peuplements d'oiseaux (fig. 2). Peu d'espèces sont capables de se maintenir tout au long d'une révolution allant d'une coupe à blanc-étoc à une vieille futaie. Ce sont les espèces ubiquistes, telles que le pouillot véloce, la fauvette à tête noire, le troglodyte et le merle en Grésigne, et encore à des degrés divers. "La plupart des espèces n'habitent donc la futaie que pendant une séquence relativement courte de son évolution entre deux coupes"(Ferry et Frochot, 1974). Dès l'apparition de la lande à éricacées puis à sarothamnes quelques années seulement après une coupe rase, la colonisation des deux espèces pionnières (fauvette grisette et hypolaïs polyglotte) est immédiate. Les coupes 


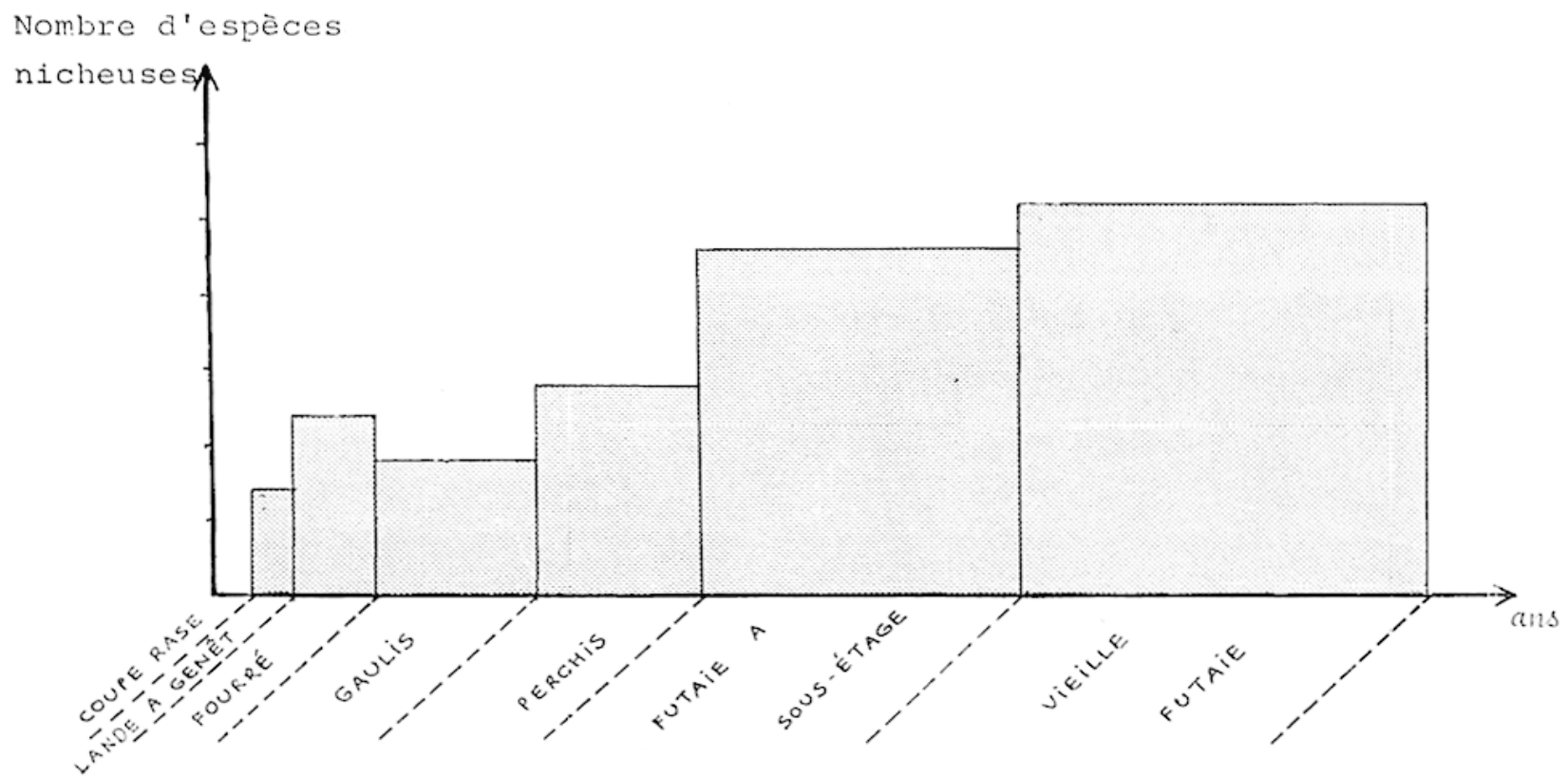

FiG. 2

Evolution de la diversité de l'avifaune nicheuse en forêt domaniale de Grésigne en fonction des stades de croissance (axe des ordonnées gradué de 5 en 5)

rases localisées au centre de la forêt domaniale de Grésigne constituent des îlots biogéographiques de superficie assez faible, correspondant généralement à une, deux ou trois parcelles (c'cst-à-dire entre 20 et 50 ha). Ces îlots sont souvent éloignés de quelques kilomètres des autres formations végétales similaires qui se situent en dehors du complexe forestier. Cet isolement relatif et ce cloisonncment des coupes peuvent expliquer leur non colonisation par des espèces indicatrices de formations herbacées ouvertes (traquet pâtre, bruants sp., chardonneret) ainsi que par certaines espèces des formations ligneuses basses ouverte (linotte) ou fermée (fauvette pitchou). Tous ces oiseaux, y compris la fauvette pitchou, rare en Grésigne, nécessitent souvent des horizons dégagés. Les coupes à blanc colonisées par la lande sont donc un milieu relativement pauvre en espèces (P12), les oiseaux les plus caractéristiques étant la fauvette grisette et l'hypolaïs polyglotte, très abondants dans l'ensemble du massif de Grésigne et donc plus aptes à la colonisation. Il en est de même pour le busard Saint Martin et l'engoulevent qui ont un assez large rayon d'action. Parmi les stades de croissance intermédiaires, les fourrés accueillent une plus grande diversité d'espèces nicheuses (P13), avec notamment l'apparition de la mésange charbonnière et des turdidés (merle noir, grive musicienne, rouge-gorge, rossignol). Par contre, les gaulis et perchis, composés de longues et jeunes «tiges", offrent peu de génotopes favorables, notamment pour les espèces cavicoles. La plus grande richesse qualitative du peuplement d'oiseaux 
est atteinte dans la vieille futaie avec un sous-étage peu dense (P1), les espèces les plus caractéristiques étant les trois pics du genre Dendrocopos, la bécasse, les mésanges bleue et nonnette, le pouillot siffleur, le gros-bec... En forêt domaniale de Grésigne, les modes de traitements entretiennent des futaies régulières. La futaie à sous étage (P3) et la vieille futaie plus ou moins claire des grands versants (P1) ont chacune un nombre d'espèces à peu près identiques, mais avec des espèces différentes (fig. 2). En futaie régulière, homogène, l'avifaune est peu variée d'une parcelle à l'autre, mais se modifie au cours de la croissance de la futaie.

\section{Conséquences de l'abandon des terroirs des causses sur l'avifaune.}

Les causses du nord et de l'ouest de Grésigne forment un complexe économiquement marginalisé qui a connu un fort exode rural depuis la fin du XIX" siècle et surtout depuis la deuxième guerre mondialc. La déprise humaine entraîne un abandon des parcelles cultivées et des pâtures, donc une évolution vers la "fermeture » des gćofaciès. L'homogénéisation qui s'ensuit fait notamment disparaître de nombreux écotones puisque les lisières finissent par se rejoindre.

Comparer les peuplements d'oiseaux de géofaciès liés à la déprise et ayant des stades d'évolution différents, permet d'émettre l'hypothèse suivante. La déprise agro-pastorale aurait pour conséquence un appauvrissement qualitatif et quantitatif de l'avifaune des milieux artificialisés et morcelés. Il y a, à cela, plusieurs raisons qui sont liées entre elles.

L'uniformisation des géofaciès entraîne une disparition progressive des écotones, d'où une diversité moindre de l'avifaune. L.a diminution des importantes potentialités alimentaires, liée à celle des trophotopes et à leur diversité (cultures céréalières, vignoble, prairies de fauche et pâtures...) entraîne le départ de nombreuses espèces granivores. d'où une réaction en chaîne sur les prédateurs. De même, la disparition des pâtures, des parcours et des troupeaux d'ovins conduit-elle à une régression des oiseaux insectivores, en particulier ceux qui se nourrissent d'insectes coprophages (huppe, étourneau, bergeronnette grise...). Enfin, on assiste à la disparition de nombreuses espèces " synanthropes " (5) et de celles qui sont directement liées à l'action humaine (moineaux sp., hirondelles sp., corvidés et notamment la pie...).

Autour de la forêt de Grésigne et notamment sur les causses de Bruniquel, Penne et Vaour, l'exode rural entraîne donc le " déménagement » ou l'exode de nombreuses espèces, en particulier de celles qui bénéficiaient le plus de l'anthropisation des milieux.

Par contre, d'autres avifaunes sont favorisées par l'exode rural et l'enfrichement des parcelles : ce sont d'abord l'avifaune des friches.

(5) Se dira d'une espèce vivant aux côtés de l'homme, notamment dans les bâtiments; le terme nous paraît plus exact qu'anthropophile. 
ensuite l'avifaune forestière. L'avifaune des milieux ouverts, anthropisés est progressivement remplacée par celle des friches, quelquefois de façon durable (dans les géofaciès ayant atteint un stade plus ou moins stable, paraclimacique), le plus souvent de façon provisoire en attendant que l'avifaune des friches soit à son tour remplacée par certains peuplements d'oiseaux forestiers. Il serait intéressant de vérifier dans l'avenir ce scénario d'évolution à partir de la connaissance des stades actuels des géofaciès.

Si l'évolution des avifaunes est dûe en grande partie aux interventions humaines, ce n'est pas toujours le cas. En effet, deux récentes catastrophes naturelles ont modifié la répartition et la composition de certains peuplements d'oiseaux en Grésigne depuis le printemps 1982. La tempête des 6 et 7 novembre 1982 a provoqué la chute de plus de $40000 \mathrm{~m}^{3}$ de bois en forêt domaniale, certaines parcelles étanı dévastées à $90 \%$, avec des répercussions sensibles sur les peuplements d'oiseaux. Les châblis ont eu des conséquences défavorables pour l'avifaune forestière, les oiseaux ćtant privés de leurs sites de reproduction et d'alimentation; par contre, les trouées favorisant le développement des formations ligneuses basses et denses avec des plantes héliophiles telles que bruyères, sarothamnes, arbustes frutes. cents, ont été progressivement colonisées par les peuplements d'ojseaux liés aux friches et aux landes. La vague de froid de janvier 1985 a entraîné une diminution sensible des effectifs de certaines espèces (rouge-gorge, fauvette à tête noire), voire la disparition totale d'espèces ayant récemment colonisé la région grésignole : c'est le cas notanment de la bouscarle de Cetti et surtout de la cisticole (apparue au début des années 1970), espèces laté-méditerranéennes particulièrement sensibles aux rigueurs de l'hiver.

\section{Conclusion}

Telle qu'elle a été tentée en Grésigne, la cartographie ornithogéographique permet seulement de situer les peuplements d'oiseaux dans un espace donné et à un moment donné, donc sans réelle possibilité de généralisation autre que méthodologique. Par contre, la valeur heuristique de la méthode ne peut faire de doute. Il faut donc $y$ voir la première phase d'un long processus de mise en place d'un observatoire de l'avifaune en fonction des changements écologiques et socio-économiques. D'autres participations scientifiques, d'autres moyens matériels ainsi que d'autres techniques, en particulier en cartographie assistée par ordinateur, seront nécessaires. En proposant ce type de cartographie ornithologique et en faisant systématiquement appel à la connaissance des milieux et à leur histoire, le géographe propose une méthode concrète pour appréhender la combinatoire entre un système, le géosystème, et un élément de ce système, l'oiseau. 
\begin{tabular}{|l}
\hline L'AVIFAUNE DANS LES GÉOSYSTĖMES DE GRÉSIGNE (TARN) : ESSAI DE CARTOGRAPHIE ORNITHOGÉOGRAPHIQUE \\
\hline
\end{tabular}

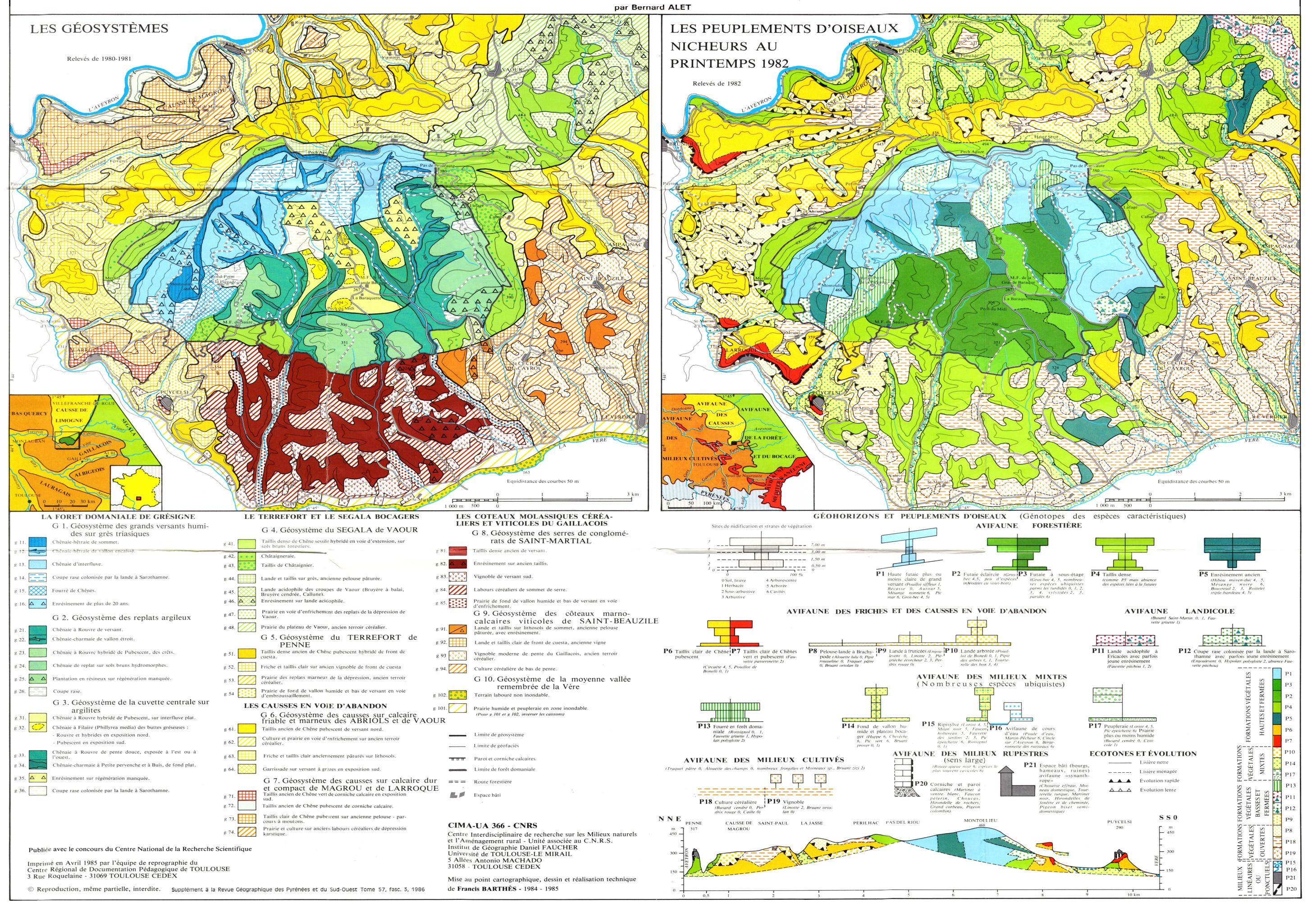




\section{Références bibliographiques}

Alet B., Andrès M., Bertin M., Pichel C. et Viglié B., (1980), Pays et paysages grésignols, Université de Toulouse-Le Mirail, 200 p. + XX p. + 6 cartes h. $t$. en couleurs au 1/25000' (Maîtrise de géographie).

Alfis B. et Bi:Rtin M., (1981), Les géosystèmes de Grésigne, Université de Toulouse-Le Mirail, 120 p. + 1 carte h. t. en couleurs au $1 / 25000^{\circ}$ (DEA géographie).

Al.:t B., (1984"), L'avifaune dans les géosystèmes de Grésigne (Tarn). Essai de carlographie ornithogéographique, Université de Toulouse-Le Mirail, 278 p + 1 pl. h. t. en couleurs (Thèse de $3^{\circ}$ cycle géographie).

Ali:r B., (1984"), Les oiseaux nicheurs en Grésigne (Tarn), RGPSO, 55, 4, pp. $392-395$.

Bi:rtrand G., (1968), Paysage et géographie physique globale. Esquisse méthodologique, $R G P S O, 39,3$, pp. 249-272.

Bertraid G., (1972), Les structures naturelles de l'espace géographique. L'exemple des montagnes cantabriques centrales, RGPSO, 43, 2, pp. 175-206.

BeroltchachVili N. et RadvaNyi J., (1978), Les structures verticales des géosystèmes, RGPSO, 49, 2, pp. $181-198$.

Beroutchachilil N. et Bertrand G., (1978), Le géosystème ou système territorial naturel, $R G P S O, 49,2$, pp. 167-180.

Blondel J., (1979), Biogéographie et écologie, Paris, Masson, 173 p.

Blondel J. et Huc R., (1978), Atlas des oiseaux nicheurs de France et biogéographie ecologique, Alauda, 46, pp. 107-129.

HANssoN L., (1977), Landscape ecology and stability of populations, Landscape planning, 4 (1), pp. 85-94.

FERRY C. et Frochot B., (1974), L'influence du traitement forestier sur les oiseaux, in Pesson P. et coll., Ecologie forestière, pp. 309-326, Paris, Gauthier-Villars.

Odum E.P., (1969), The strategy of ecosystem development, Science, 164, pp. 262. 270.

Yeatman L., (1971), Histoire des oiseaux d'Europe, Paris, Bordas, 368 p.

YeatMan L. et coll., (1976), Atlas des oiseaux nicheurs de France de 1970 à 1975 , Paris, Société ornithologique de France, $282 \mathrm{p}$. 
Résumé. - La méthode géosystémique, appliquée à l'ornithologie, resitue les peuplements d'oiseaux dans l'espace et dans le temps et leurs stratégies d'utilisation du milieu; elle peut contribuer à une cartographie intégrée de géographie ornithologique. La difficulté principale est d'intégrer les oiscaux dans un milieu défini, en raison de leur mobilité et de leurs exigences spatiales très changeantes au fil des saisons, sauf au printemps, en période de reproduction; aussi la carte des peuplements ne prend-elle en compte que les oiseaux nichcurs. $\mathrm{La}$ carte des géosystèmes et celle des peuplements d'oiseaux nicheurs sont ensuite comparées avec leurs techniques respectives de réalisation. Il existe plusicurs avifaunes correspondant aux quatre complexes géographiques mis en évidence. A ces quatre grandes avifaunes, s'ajoutent des avifaunes ou peuplements localisés, liés à des habitats peu étendus ou marginaux. Chaque peuplement d'oiseaux est étudié dans son avifaune. L'analysé géosystémique permet de comprendre l'effet et les conséquences de l'action anthropique sur l'évolution des géosystèmes et de leur avifaune. Les oiseaux, caractéristiques d'un état du géosystème à un moment donné, sont des témoins de cette évolu. tion et sont à ce titre des indicateurs fiables parce que sensibles aux transformations de leur milieu de vie. Tout géofaciès constitue un stade intermédiaire, provisoire, qui explique le caractère éphémère des peuplements d'oiseaux mais aussi leur caractère répétitif au cours du temps. La cartographie ornitho-géographique, représentant les peuplements d'oiseaux à un moment donné, dans un espace donné, doit être réactualisée pour suivre l'évolution de ces peuplements dans l'espace et dans le temps.

Summary. - Birds aNd the geosystem : Mapping birdforms in the Grésigne MasSIF (Tarn). The reconstruction in space and time of bird populations, as well as their environment use strategies is made possible by the application to ornithology of the geosystemic method. This contributes to an integrated cartography of geographic ornithology. It is uneasy to link birds to a defined environment, due to their mobility and their changing seasonal spatial demands, except in spring, with reproduction: therefore the population map only refers to nesting birds. The geosystem map and that of birds are then compared. There are several distinct avifauna, linked schematically to the four geographic complexes indicated by the map of geosystems. Other avifauna and local populations are linked to limited or marginal habitats. Each bird population being studied in relation to its avifauna, the geosystemic analysis allows to understand the consequences of human action on the evolution of geosystems and their avifauna. The birds, characteristic of a certain state of the geosystem, indicate this evolution. Each basic landscape structure constitutes an intermediate stage which explain the ephemeral nature of these bird populations, but also their respective nature in time. The ornitho-geographic cartography, which represents the bird population at a moment in space and time, must be regularly recreated in order to follow their evolution in space and time.

Resumen. - Las aves dentro del geosistema. Cartografía de la avifalina en fi. macizo dE Gresigne (Tarn). El método geosistémico aplicado a la ornitología permite situar las poblaciones de aves en el espacio y en el tiempo así como conocer sus estrategias de aprovechamiento del medio ambiente, contribuyendo así a una cartografía integrada de geografía ornitológica. La principal dificultad es la de integrar las aves en determinados medios a causa de su movilidad y de sus exigencias espaciales muy variables según la estación. Por eso el mapa de las poblaciones sólo toma en cuenta las aves anidadoras. Después se compara este mapa de aves con el de los geosistemas. Resulta que no hay una, sino diversas avifaunas que coinciden esquemáticamente con los cuatro complejos geográficos puestos en evidencia por el mapa de los geosistemas. A la cuatro avifaunas principales se añaden otras locales que corresponden a habitats de poca extendidos o marginales. Cada población de aves es estudiada dentro de la avifauna a la que pertenece. Con el análisis geosistémico también se puede entender el impacto de la acción antrópica en la evolución de los gcosistemas y de sus avifaunas. Las aves dan fe de esta evolución porque son sensibles a las modificaciones de su entorno vital. Cualquier geofaciés es un estado intermedio y provisional que explica el carácter efímero de las poblaciones de aves y también su carácter repetitivo en la Historia. De allí la necesidad de una permanente reactualización de la cartografía ornitogeográfica.

Mots-CI.És. - Bassin aquitain, Tarn, GrÉsIGNe, zoogéographie, ornithogéographie, avifaune, peuplement d'oiseaux, indicateur écologique, écologie historique, chorologie, phénologie, géosystème, géofaciès, géohorizon, génotope, trophotope, écotone, cartographie ornitho-géographique, actuel. 